Martins, M.J., Castilho, P., Santos, V., \& Gumley, A. (2016). Schizophrenia: An Exploration of an Acceptance, Mindfulness, and Compassion-based Group Intervention. Australian Psychologist, 52(6), 514-523. doi: 10.1111/ap.12210.

\title{
Schizophrenia: An Exploration of an Acceptance, Mindfulness, and Compassion-based Group Intervention
}

Maria João Martins, ${ }^{1,2}$ Paula Castilho, ${ }^{1}$ Vitor Santos, ${ }^{2}$ and Andrew Gumley ${ }^{3}$

${ }^{1}$ Faculty of Psychology, University of Coimbra, ${ }^{2}$ Community Mental Health Team, Baixo Vouga Hospital Centre, and ${ }^{3}$ Institute of Health and Wellbeing, University of Glasgow

Objective: This study aimed to develop and apply a brief (five-session) group-based intervention called Compassionate, Mindful and Accept- ing approach to Psychosis (CMAP) for patients diagnosed with paranoid schizophrenia. Methods: The intervention was based on three major approaches: the mindfulness framework adapted for psychosis with the proposed modifications for meditation work, the rationales from Acceptance and Commitment Therapy, and Compassion-Focused Therapy adapted to psychosis. The intervention was in group format, with one therapist (five 1-hr sessions). Five patients (male, single, between 22 and 35 years old, Caucasian) completed the intervention. Participants completed self-report measures at baseline (1 week prior to intervention) and post- treatment ( 1 week-additionally the Satisfaction with Intervention Questionnaire). Results: The intervention seemed acceptable for all participants. For illustration of potential benefits of this approach, pre-post results are presented and discussed for two patients. Overall, there was improvement in both patients, although in different measures. Both patients' conviction in paranoid delusions decreased, while an increase in acting with awareness was observed.

Conclusions: Although preliminary, the results are in line with previous research in psychosis. Future directions and clinical implications are discussed.

Key words: Acceptance and Commitment Therapy; Compassion-Focused Therapy; mindfulness; schizophrenia. 


\section{What is already known on this topic}

1 Contextual therapies aim at developing a more flexible response and more useful self-others and self-self relationships rather than eliminating symptoms.

2 Acceptance, mindfulness, and compassion-based therapies for psychosis have shown promising results in several outcomes.

3 To our knowledge, only one integrated treatment combining CAM exists and found promising results regarding acceptability, self-regulation, and affective symptoms.

\section{What this topic adds}

1 This study aimed at developing a brief and easy to implement intervention based on acceptance, mindfulness, and compassion for schizophrenia.

2 Understanding of acceptability in sample of five patients: intervention seemed acceptable with subjective improvement of difficulties and coping.

3 Potential benefits of this approach are illustrated through the results of two case studies: improvement in paranoid conviction and acting with awareness.

Contextual cognitive behavioural therapy (CCBT; Hayes, Villatte, Levin, \& Hildebrandt, 2011) emerged as a response to criticisms of the cognitive behaviour therapy model, where the function, not content per se, of internal events (including thoughts and images) were regarded as key to understanding patterns of psychological distress (Barlow, 2002). Based on this, contextual therapies embrace a series of methods that emphasise developing an accepting attitude towards internal events, emphasising their context and function regardless of the logical or evidential content. Therefore, the main goal of contextual therapies is developing a flexible repertoire of functional behavioural responses to internal experiences rather than the elimination of specific symptoms. Emphasis is placed on values, quality of life, and acceptance of internal experiences in the "here and now" through experiential exercises (e.g., mindful- ness). There are several approaches with the principles of the contextual therapies, such as mindfulness-based therapies, Acceptance and Commitment Therapy (ACT; Hayes, Luoma, Bond, Masuda, \& Lillis, 2006), and Compassion-Focused Ther- apy (CFT; Gilbert \& Procter, 2006). 
In psychosis, the focus on contextual strategies may be beneficial where contextual therapies focus on modifying the per- son's relationship with their experiences (e.g., hearing voices) (Bach \& Hayes, 2002), facilitating a therapeutic process of engaging flexibly with psychotic experiences (Gaudiano \& Her- bert, 2006). For example, Chadwick, Newman-Taylor, and Abba (2005) have emphasised the role of mindfulness strate- gies in supporting individuals becoming aware of their psy- chotic experiences as impermanent and distinct from self.

\section{CCBT for Psychosis: From Case Reports to Randomised Controlled Trials}

\section{Acceptance and Commitment Therapy}

Single-case data have showed promise in utilising acceptance-based strategies in psychosis. ACT intervention has also been associated with reduction of frequency of auditory hallucinations (García-Montes \& Pérez-Álvarez, 2001), delusional verbalisations (García-Montes, Luciano, Hernández, \& Zalvivar, 2004), symp- tom believability and distress (Pankey \& Hayes, 2003), and nega- tive symptoms (García-Montes \& Pérez-Álvarez, 2010). These case studies’ results also showed promising behaviour outcomes improvement as rated idiosyncratically with the patient (Pankey \& Hayes, 2003) and increases in valued action (García- Montes \& Pérez-Álvarez, 2001; García-Montes et al., 2004). Importantly, these case studies showed that ACT was feasible and acceptable with individuals experiencing psychosis.

To date, five RCTs have been published on ACT or acceptance-based interventions for psychosis (Bach \& Hayes, 2002; Gaudiano \& Herbert, 2006; Gaudiano et al., 2015; Shawyer et al., 2012; White et al., 2011). In a study with 80 participants with positive psychotic symptoms, randomised to "Treatment As Usual" (TAU) and TAU plus four individual sessions of ACT, Bach and Hayes (2002) found that ACT was linked to significantly higher symptom reporting (consistent with reduced avoidance), lower symptom believability, and reduced re-hospitalisation over a 4-month follow-up period. Gaudiano and Herbert (2006), with participants being ran- domly assigned to enhanced treatment as usual (ETAU) or ETAU plus three (on average) individual sessions of ACT, found that ACT was associated with reduced self-rated distress related to hallucinations and social disability. Data from this trial were further examined by Gaudiano, Herbert, and Hayes (2010), and believability of hallucinations at post-treatment was found to mediate the effect of ACT on hallucination- related distress. Bach, Gaudiano, Hayes, and Herbert (2012), combining the data from the two trials described above (Bach \& Hayes, 2002 and Gaudiano \& Herbert, 2006), con- cluded that the reduction in hospitalisation rates was improved for ACT in intent to treat analysis and 
that decreased believability in the content of symptoms was related to reduced re-hospitalisation at 4-month follow-up. White et al. (2011) found that ACT (14 patients with 10 one-to-one sessions) was associated with greater improvement in negative symptoms, fewer cases of depression, and a significant increase in mindfulness skills. Increasing mindfulness over time was correlated with reducing depression. Shawyer et al. (2012) found no effects for ACT (15 individual sessions) regarding confidence to resist harmful commands or in ability to cope with them. However, only $41 \%$ of the sample reported compliance to harmful command hallucinations at baseline, weakening the power to detect effects in the primary outcomes. No significant differences were observed between the groups in any of the outcomes (i.e., changes in illness severity, better functioning, reduction in distress, or improve- ment of quality of life).

Finally, Gaudiano et al. (2015) found that ACT (16 individual sessions) was associated with improved depression in people with psychosis as well as psychosocial functioning (measured by the World Health Organization Disability Assessment Schedule) and experiential avoidance. Small effect sizes were also found for psychotic symptoms.

\section{Mindfulness-based Interventions}

Case studies have testified to the feasibility and acceptability of mindfulness for psychosis (Jacobsen, Morris, Johns, \& Hodkin- son, 2011; Newman-Taylor, Harper, \& Chadwick, 2009), and further evidence from grounded theory analyses have testified to service users' engagement with the experience of mindful- ness (Abba, Chadwick, \& Stevenson, 2008). Two feasibility studies have found evidence of acceptability in first episode of psychosis (Van der Valk, Van de Waerdt, Meijer, Van den Hout, \& de Haan, 2013) and improved anxiety, depression, and conviction in and preoccupation with delusions as well as improvements in processing speed and working memory (Tabak \& Granholm, 2014).

Four RCTs have been conducted on mindfulness for psycho- sis (Chadwick, Hughes, Russel, Russel, \& Dagnan, 2009; Chien \& Lee, 2013; Chien \& Thompson, 2014; Langer, Cangas, Salcedo, \& Fuentes, 2012)

Chadwick et al. (2009) demonstrated feasibility of randomising individuals to mindfulness groups (10 sessions) and acceptability of mindfulness itself. Although there were no differences between groups, improvements were observed in clinical functioning (conceptualised as subjective well-being, problems and symptoms, life functioning, and risk) and mindfulness of distressing thoughts and images. Langer et al. (2012) found that no significant effects were observed in any measure between the groups, except in mindfulness response to stressful thoughts and images 
within the Mindfulness-Based Cognitive Therapy group (eight individual sessions). Chien and Lee (2013) found that Mindfulness-based Psychoeducation (MBP-12 group sessions) was associated with significant change in symptom sever- ity, illness insight, and length of re-hospitalisation at post- intervention, while functioning and number of re- hospitalisations improved significantly only at the 18-month follow-up. Chien and Thompson (2014) found that MBP was associated with greater improvement in insight and treatment attitudes, functioning, psychiatric symptoms, and duration of hospital readmissions.

\section{Compassion-Focused Therapy}

In a single case series, Mayhew and Gilbert (2008) found that CFT was feasible and acceptable and that participants showed decreases in depression, psychoticism, anxiety, obsessive-compulsive symptoms, paranoia, and interpersonal sensitivity at post-inter- vention. Laithwaite et al. (2009) found improvements associated with a compassion-focussed intervention (20 group sessions) in terms of social comparisons, shame, depression, and self-esteem. Loving kindness meditation (six group sessions) was found to be feasible and associated with decreased negative symptoms and increased positive emotions and psychological recovery (Johnson et al., 2011).

The only RCT on CFT for psychosis (Braehler et al., 2013) found that CFT (16 group sessions) was feasible, acceptable, and not associated with adverse effects. The CFT group had greater observed clinical improvement (measure of improve- ment/exacerbation relative to baseline) and revealed higher levels of compassion, which were correlated with lower levels of depression and social marginalisation). 


\section{Integrated Treatment Approaches}

Khoury, Lecomte, Comtois, and Nicole (2013) developed an integrated treatment for emotional regulation combining contextual strategies, namely compassion, acceptance, and mindfulness (CAM), for individuals with early psychosis and found promising results regarding acceptability, feasibility, and potential clinical utility of this approach. Improvements were found in emotional selfregulation (e.g., less rumination, catastrophisation).

Based on these findings, our study aimed to continue the empirical work that has been done in terms of CCBT approaches for psychosis. Therefore, the present preliminary and explorative study aimed to develop a five-session group intervention called Compassionate, Mindful and Accepting approach to Psychosis (CMAP). The way C.MAP differs from other intervention proto- cols (and is similar to the Khoury et al. intervention) is that C. MAP intends to include different interrelated processes that have been studied as potentially effective in psychosis instead of focus- ing on one specific therapeutic approach that can maximise the therapeutic gains. Our main goal was not to prove efficacy of the C.MAP but to (a) develop a brief intervention with the potential for routine implementation in outpatient contexts, (b) explore the potential benefits of this brief intervention, and (c) understand the way participants experience these new strategies. Our hypotheses were:

- Participants would positively evaluate the C.MAP in terms of usefulness in improving difficulties, perceived coping with difficulties, usefulness of handouts, intention to participate in other groups, perceived ease of mindfulness exercises, and intention to use the exercises in the future through an evaluation questionnaire;

- Participants would show lower levels of paranoid ideation, shame, self-criticism, and would report higher levels of acceptance and mindfulness.

\section{Methods}

\section{Participants}

Ethical approval was obtained from the Baixo Vouga Hospital Centre ethics committee prior to the study. The participants were identified and referred for the intervention by their psychiatrist (in a Community Mental Health Team) and gave informed consent (Declaration of Helsinki) after a meeting with the principal researcher, where objectives and roles were clarified. Inclusion criteria were: (a) diagnosis of schizophrenia (made by each participant's psychiatrist), (b) absence of significant cognitive deficits, (c) clinical stabilisation (stable residual symptoms were permitted), (d) 
aged above 18, and (e) outpatients. Exclusion criteria included severe positive or negative symptoms or severe cognitive deficits as identified and informally assessed by each participant's psychiatrist prior to inclusion in the study. Seven patients showed interest and fulfilled criteria; all were male, Caucasian, aged between 22 and 35 years old $(M=27.86$; DP $=5.15)$, had 5-12 years of school education $(M=8.57$; DP $=2.37)$, and were of low to medium socioeconomic status (calculations based on family monthly income). The participants had experienced between zero and five hospitalisations. All of the patients had substance (mainly cannabis) abuse in the past, but only one was current. Three participants were employed. One participant withdrew from the study prior to the first session, and one participant dropped out after session two (unknown reasons). Data regarding programme acceptability is presented for all participants who completed the programme. However, we only present outcome data as illustrative of potential intervention benefits for two participants. This decision was made after the first assessment because three of the participants had difficulties in completing the questionnaires in a valid manner. Problems were detected concerning two main aspects: (a) social desirability: some patients minimised their difficulties (based on known characteristics of this population, we can hypothesise that this may have occurred due to avoidance or stigma-related aspects) and (b) albeit a researcher being present during the assessment, possible cognitive deficits and/or difficulties in abstract thinking seem to have influenced responses from some patients as they did not present a congruent pattern, particularly in more complex questionnaires (e.g., contradictory answers). Moreover, the face-to-face assessment might have been threatening for these patients, and the validity of responses might have been affected by shame-related and interpersonal difficulties.

The two participants had different clinical presentations. Participant 1 was a 22-year-old male, single, unemployed, 5 years of education, living with an aunt. He was first diagnosed with paranoid schizophrenia at age 19 and had two hospitalisations, the first due to persecutory delusions and conceptual disorganisation and the second (compulsory) due to ideas of reference, persecutory delusions, disorganised speech and behaviour. The pharmacological treatment included clozapine 150 mg day ${ }^{-1}$ and diazepam $10 \mathrm{mg} \mathrm{day}^{-1}$. Participant 2 was a 31 -year-old male, single, unemployed, 9 years of education, living with his parents. He was diagnosed with paranoid schizophrenia at the age of 19 . He had one hospitalisation. The participant had residual positive (auditory hallucinations) and negative symptoms (predominant) and severe anxiety. The past delusional activity had mystical content. The pharmacological treatment was risperidone $6 \mathrm{mg} \mathrm{day}^{-1}$; paroxetine $20 \mathrm{mg}$ day $^{-1}$, and lorazepam $5 \mathrm{mg} \mathrm{day}^{-1}$.

\section{Measures}


Participants completed an assessment battery of self-report measures at the baseline (1 week prior group therapy) and at post-treatment (1 week after). Internal consistencies were cal- culated at pre-test $(N=6)$, and as we were working with a small sample size, our alpha coefficients were overall low; therefore, we decided to accept alphas higher than .50.

\section{Paranoia checklist (PC, Freeman et al., 2005)}

This 18 -item scale was devised to investigate paranoid thoughts of clinical populations. The participants were asked to rate each item on a 5-point scale for frequency, degree of conviction, and distress. Higher scores indicate higher levels of frequency, con- viction, and distress associated with paranoid thoughts. In the original study, the results showed excellent internal reliability: .90 or above for all subscales. In the Portuguese validation study, the subscales' alphas were .92 (frequency) and .95 (con- viction and distress) (Lopes, 2010). In the present study, the internal consistencies ranged from .58 to .81 .

\section{Other as Shamer Scale (OAS, Goss, Gilbert, \& Allan, 1994)}

The scale consists of 18 items rated on a Likert 5-point scale according to the frequency of evaluations about how others judge the self. Higher scores are indicative of higher levels of external shame. The scale showed high internal consistency - .92. A short version (6-item) was developed (Matos, Pinto-Gouveia, Gilbert, Duarte, \& Figueiredo, 2015) and it also revealed an excellent internal consistency $(\alpha=.91)$ and temporal stability $(r=.70)$. In the present study, the internal consistency was .88.

Functions of Self-Criticism and Reassuring Scale (FSCRS, Gilbert, Clarke, Hempel, Miles, \& Irons, 2004)

This 22-item scale was developed to assess people's critical and reassuring self-evaluative responses through a 5-point Likert scale. Factor analysis suggested three subscales with excellent internal consistencies (alphas ranging from .86 to .90). The Portuguese version (Castilho \& PintoGouveia, 2011) revealed the same structure, with alphas ranging from .62 to .89. We combined the “inadequate" and "hated self” subscales into a self- criticism score, with higher scores meaning higher levels of self-criticism. In the self-reassurance scale, higher scores mean higher levels of selfreassurance skills. The alphas for self- criticism and self-reassurance in this study were .85 and .58 .

Acceptance and Action Questionnaire-II (AAQ-II, Bond et al., 2011)

This 7-item self-response questionnaire aims to assess psychological acceptance and experiential avoidance (on a 1-7 rating scale), with higher scores indicating lower levels of 
psychological flexibility. Results indicated satisfactory structure, reliability (.78-.88), and validity. The Portuguese unifactorial version showed excellent internal consistency (.90) and good convergent and discriminant validity (Pinto-Gouveia, Gregório, Dinis, \& Xavier, 2012). In this study, the internal consistency was .87 .

Five Facets of Mindfulness Questionnaire (FFMQ, Baer, Smith, Hopkins, Krietemeyer, \& Toney, 2006)

This 39-item self-report instrument comprises five facets: observing, describing, acting with awareness, non-judging of inner experience, and non-reactivity. Higher scores in each facet reflect higher levels of the correspondent mindfulness facet. In the original study, all five facet scales showed internal consistency from .75 to .91. In the Portuguese version, the facets presented adequate internal consistency (.66-.89) (Gregório \& Pinto-Gouveia, 2011). In this study, due to low internal consistencies, only the "observing" (alpha of .90), "acting with awareness" (.70), and "non-judging" (.58) values were used.

\section{Satisfaction with Intervention Questionnaire}

This instrument was specifically designed to assess (anonymously) the experience that patients had with the group; on a 4-point Likert scale, the patients were asked to assess: their difficulties ("How do you feel your difficulties are after the pro- gram?"-1 = "Much worse" to 4 = "Much better), coping strategies ("How do you feel your ability to deal with your difficulties is after the program?"-1 = "Much worse" to $4=$ "Much better"), hand-outs ("How useful were the handouts?"- $1=$ "Not at all" to $4=$ "Very much"), intent to participate in other groups ("Do you consider participating in other groups offered in this team?"-1 = "Not at all" to $4=$ "Highly motivated"), mindfulness exercises ("How difficult did you found the mindfulness exercises?"- $1=$ "Very difficult" to $4=$ "Very easy"), and the probability of using them in the future ("What is the probability of using these exercises in the future?"- $1=$ "Not likely at all" to 4 = "Highly likely").

\section{Procedure}

Due to specific characteristics of the target population, the self- report measures were completed with the support of one researcher who assessed the validity of responses. The participants continued to benefit from psychiatric appointments throughout the study, but none of the participants had individual psychotherapy simultaneously. 


\section{Development of the intervention}

The team who developed the C.MAP integrated clinical psychologists with clinical experience in psychosocial interventions for schizophrenia and contextual cognitive-behavioural therapeutic models.

Results from other CCBT interventions for psychosis were taken into consideration, and efforts were made to accommodate recent findings and feedback from professionals in the area. The intervention was based on three major approaches: the rationales from mindfulness framework adapted for psychosis (Chadwick et al., 2005), ACT adapted for psychosis (Bach \& Hayes, 2002), and CFT (Gilbert \& Procter, 2006). The proposed modifications of mindfulness meditation to work with patients with psychosis (Chadwick et al., 2005) were made. The exercises for each session were chosen taking into account: (a) the team's psychologist's clinical experience (with CCBT forother populations and psychotherapeutic intervention-CCBT and CBT-for schizophrenia), (b) the feedback provided by professionals with expertise in CCBT for psychosis (considering the feedback from previous CCBT groups), and (c) existing intervention protocols.

The C.MAP: A Compassionate, Mindful and Accepting Approach to Psychosis

This intervention focused on developing a more adaptive and functional way to respond to psychological experiences including psychotic symptoms through developing mindfulness skills and cultivating compassionate and accepting responses to thoughts and behaviour. A brief outline and detailed information on the sessions is presented in Table 1. 
Table 1.

Intervention Outline

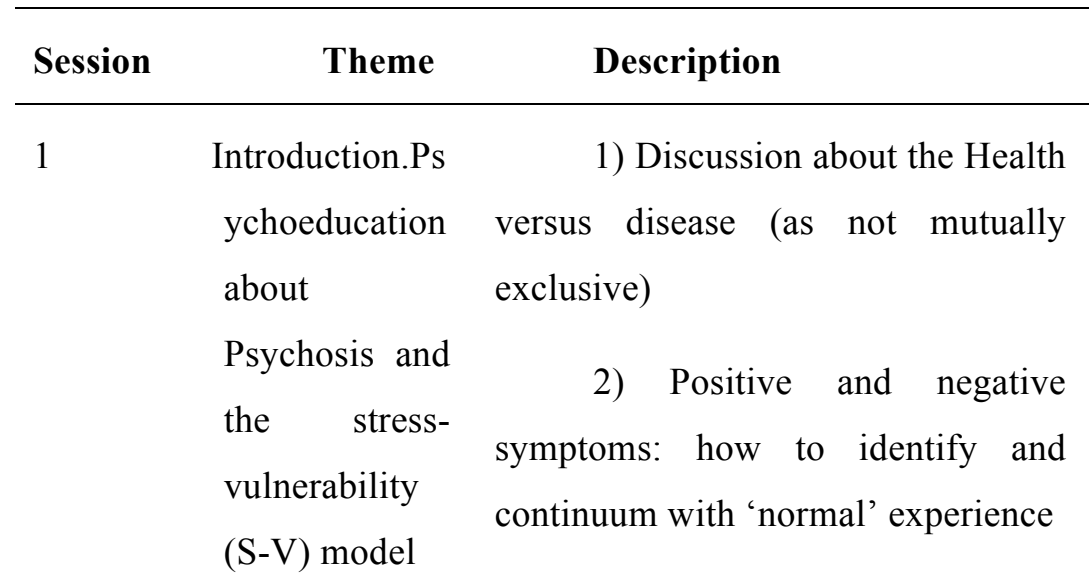

3) Other symptoms associated with psychosis (anxiety, shame, depression, among others)

4) S-V model: understanding multiple causes for psychosis (deshaming)

5) Introducing the intervention

- Group exercise "Getting to know each other"

- Filling in the "Health and Disease Circle" at different stages of life (percentages of health and illness)

- Discussion of the "Where from and how its maintained" Handout (predisponents, precipitants and maintenance factors of psychosis)

- Discussion (at this stage sharing personal experiences was not required although some participants did; several examples were made available) as a different way of dealing with symptoms and their interference on everyday life, as well as a form of relapse prevention. 
Mindfulness. "automatic pilot" mode

like to do?"

2) Cognitive fusion and

moment.

experiential avoidance versus

experiential acceptance and

willingness

3) Introducing Mindfulness:

definition, what Mindfulness is and is

not.

3 Acceptance vs

Experiential

Avoidance.

Acting with

commitment thoughts' experiences
- Discussion on the 'un-controllability of

- Experiential exercise: Yellow Jeep and sharing experiences

- Experiential exercise: Mindfulness of breath (5 minutes) and sharing

- Experiential exercise: "Being willingly out of breath" and sharing experiences

- Experiential exercise: Mindfulness of Breath (5 minutes)

2) Practicing acceptance - Experiential exercise: "Supressing an unwanted thought" and sharing experiences

3) Mindfulness meditation

4) Life Directions
- Mindfulness of Breath (3 minutes)

(3minutes)

- "Making a small
change": participants were encouraged to take a small action they were afraid of because thoughts and emotions suffering went away" handout and Discussion (introducing acceptance as an alternative)

- Experiential exercise: Mindfulness of emotions (where does the anxiety feel in our body?) (3 minutes) 
- Experiential exercise: Mindfulness of thoughts (allowing an unwanted thought to exist) (3 minutes) sharing experiences
2) 3 affect regulation systems and their outputs

3) Self-critical versus selfcompassionate thoughts

4) Loving-kindness meditation

- Experiential exercise: Mindfulness of breath (3 minutes) + Loving Kindness meditation (compassionate wishes towards the self) and sharing experiences

1) What is relapse and strategies for relapse prevention

2) Risk and relapse signs

3) Mindfulness meditation
4) Evaluation
of the

Experiential exercise: Mindfulness of Breath (5 minutes)

- Group Exercise: “Compassionate Mind versus Critical Mind" (cards with thoughts from both minds to discuss and identify the corresponding mind for each thought)

Experiential exercise: Mindfulness of Breath (5 minutes) and sharing experiences

- Filling in the "My warning signs" and "What to do when Relapse starts to show?" handouts and Discussion intervention
Mindfulness of Breath (3 minutes)

\section{Loving-kindness}

exercise practiced in the

- Experiential exercise: Mindfulness for 
stress (imagining a stressful situation)

with loving-kindness (compassionate

wishes for the self) and sharing

experiences 
The intervention was delivered in a closed-group format, and the group was planned to have a minimum of five participants and a maximum of eight, according to specific recommendations for running group interventions with psychosis (Braehler et al., 2013). Also, considering the abovementioned recommended guidelines, the five weekly sessions had a duration of $1 \mathrm{hr}$ (with a 5min break); all sessions followed the same struc- ture to reduce anxiety (informal welcome, session theme with exercise, sharing experiences, summary, and introduction to homework), and exercises were brief (3-10 min). Sessions were delivered by one therapist, a clinical psychologist, with weekly supervision of a senior therapist. At the end of each session, hand-outs were provided, and patients were encouraged (although homework was not mandatory) to practice between sessions. Patients were provided with the exercise script, and a family member was chosen to read the script and help with the practice at home.

\section{Results}

\section{Acceptability Results}

Overall, the intervention was well tolerated by all participants who completed the intervention $(n=5)$, and four reported improved perception and ways of dealing with difficulties. All five completers reported to be willing to take another group intervention and considered the hand-out provided "useful." Two participants found mindfulness exercises "difficult," but all patients endorsed the possibility of "using these exercises in the future."

\section{Outcome Results}

Overall, we observed that there was improvement in both participants after intervention. In order to assess the reliability and clinical significance, we used the "Reliable Change Index" statistic (RCI) developed by Jacobson and Truax (1991) (Table 2). The RCI, which provides a measure of both statistical and clinical significance taking into account the scale reliability, is useful in small sample clinical populations to assess effectiveness, with a focus on individual change (Zahra \& Hedge, 2010). We used the indications of E.A. Wise $(2004$, p. 56) for interpretation purposes: results greater than $|.84|$ (significant change), result exceeding $|1.28|$ or $|1.96|$ (remission), and 95\% (recovery). 
Table 2.

Total Scores and RCI scores for Participants 1 and 2

\begin{tabular}{|c|c|c|c|c|c|c|}
\hline & \multicolumn{3}{|c|}{ Participant1 } & \multicolumn{3}{|c|}{ Participant2 } \\
\hline & Pre & Post & & Pre & Post & \\
\hline & Score & Score & $\mathrm{RCI}$ & Score & Score & $\mathrm{RCI}$ \\
\hline \multicolumn{7}{|l|}{$\mathrm{PC}$} \\
\hline Frequency & 23 & 24 & .33 & 20 & 18 & -.66 \\
\hline Conviction & 39 & 29 & $\begin{array}{l}- \\
3.48\end{array}$ & 21 & 18 & -1.05 \\
\hline Distress & 15 & 21 & 2.42 & 3 & 0 & -1.21 \\
\hline OAS & 9 & 3 & $\begin{array}{l}- \\
3.57\end{array}$ & 0 & 0 & .00 \\
\hline \multicolumn{7}{|l|}{ FSCRS } \\
\hline Self-criticism & 21 & 21 & .00 & 5 & 2 & -1.83 \\
\hline Self-reassurance & 20 & 12 & $\begin{array}{l}- \\
3.25\end{array}$ & 7 & 32 & 10.14 \\
\hline AAQ & 27 & 18 & $\begin{array}{l}- \\
6.11\end{array}$ & 7 & 8 & .68 \\
\hline \multicolumn{7}{|l|}{ FFMQ } \\
\hline Observing & 10 & 8 & $\begin{array}{l}- \\
5.23\end{array}$ & 7 & 19 & 31.41 \\
\hline Acting with awareness & 31 & 36 & 7.40 & 36 & 37 & 1.48 \\
\hline Non-judging & 28 & 34 & 7.56 & 33 & 31 & -2.52 \\
\hline
\end{tabular}

Note: $\mathrm{PC}=$ Paranoia Checklist; $\mathrm{OAS}=$ Other as Shamer Scale; $\mathrm{SBS}=$ Submissive Behaviour Scale; FSCRS=Functions of Self Criticism and Reassurance Scale (FSCRS); AAQ=Acceptance and Action Questionnaire; FFMQ=Five Facets of Mindfulness Questionnaire 


\section{Discussion}

This study aimed to develop and apply a brief contextual intervention based on the mechanisms proposed by third- generation therapies, merging the advantages of the different intervention rationales - the C.MAP. We intended to explore the perceived usefulness of the intervention and gather the participants' opinions as well as analyse its potential benefits.

Overall, most of the participants considered the C.MAP useful and reported subjective improvement of difficulties and ways of dealing with difficulties. Acceptability results for thirdgeneration behaviour therapies have been found for ACT (e.g., White et al., 2011), mindfulness (e.g., Chadwick et al., 2009; Jacobsen et al., 2011; Johnson et al., 2011), and compassion- focused interventions (e.g., Braehler et al., 2013) in previous research. Our first hypothesis was, in this way, supported by results in spite of limitations concerning the method used to measure acceptability (e.g., a questionnaire not otherwise tested) and the sample size.

Given the very small sample size and lack of outcomes data, we are only able to consider potential treatment signals that may provide a basis for developing the intervention further. Both participants seemed to have increased the mindfulness skill "acting with awareness" over time, and this may have important benefits for developing skills to identify and respond to early warning signs of relapse (e.g., Birchwood, Spencer, \& McGovern, 2000) and to identify patterns of relationships between context, internal events, and behavioural responses. We also observed reduced paranoid conviction in both participants. This is in line with previous research (e.g., Bach \& Hayes, 2002; Gaudiano \& Herbert, 2006) and may signal participants' beginning to become less fused with their paranoid thoughts. This may be of clinical significance given that fusion with experiences may increase feelings of entrapment in psychotic experiences (e.g., Taylor et al., 2010) and its reduction may allow patients to pursue valued life goals (Hayes et al., 2006). Schizophrenia is known to have different presentations, and studies have found different patterns in response to psychosocial treatment depending on the clinical presentation (Bach \& Hayes, 2002). Also, in our study, and considering that the patients had different presentations, the results showed that, overall, there was improvement in both patients, although in different aspects. The following results will be discussed separately for each patient as different patterns of results indicate different possible interpretations and may highlight different implications. We find it useful to discuss the different results from the two patients as the clinical response to the same intervention can be different in different patients, and the reflection on different possible explanations might be useful in clinical practice. 


\section{Participant 1}

After intervention, the participant seemed more willing to be in contact with the private events without trying to avoid, alter, or suppress them (Hayes, Strosahl, \& Wilson, 2011). Reducing experiential avoidance levels is thought to be important as previous research has shown that higher experiential avoidance is associated with greater delusions (Udachina et al., 2009). Although literature recommends including mindfulness and acceptance training in clinical protocols as it could lead to clini- cal improvement both in psychotic (Chadwick et al., 2005, 2009) and post-psychotic (White et al., 2013) symptoms, the field is in need of mediational studies to better evaluate the mechanisms behind therapeutic change.

The participant also seemed to have learnt to observe inner experience in an accepting way, refraining from judgments or criticism (Baer et al., 2006), which is congruent and has the same implications as the previously mentioned results. Never- theless, the patient maintained the levels of self-criticism, with a weaker capacity to self-soothe and self-reassure in situations of failure. Although not expected, this result can be interpreted considering that self-related cognitions may be more difficult to accept (particularly with a short intervention) than other types of thoughts and experiences (e.g., other-related, world-related). The soothing system has been described as potentially underde- veloped in psychosis, and therefore, difficulties in accessing this system are common (Gumley, Braehler, Laithwaite, MacBeth, \& Gilbert, 2010). We hypothesise that the person may have gained awareness of the "threat-based mind versus soothing and compassionate mind" throughout the intervention and therefore responded in a more attuned way at post- intervention assessments.

There was a significant reduction in external shame, an important output of the threat system, as this type of shame would orientate behaviour towards safety strategies. Studies have highlighted the association between external shame and psychotic symptoms, including paranoid ideation (e.g., Matos, Pinto-Gouveia, \& Gilbert, 2013). Shame has also been shown to be associated with higher levels of social dysfunction in psy- chosis populations (Birchwood et al., 2007). Thus, the observa- tion that participant 1 's feelings of shame reduced over time may be an important treatment signal. Future research should try to understand the benefits of focusing on shame to prevent relapse and promote recovery in psychosis.

\section{Participant 2}

In Participant 2, we observed a different pattern of results, including an unexpected increase of 
experiential avoidance strategies. This result could be explained by the higher levels of dysfunctional emotional regulation strategies that have been associated with psychosis (Livingstone, Harper, \& Gillanders, 2009). This result could also be understood regarding the fear of experiencing affiliative emotions (widely studied in psychopathology; for a review, see Veale, Gilbert, Wheatley, \& Naismith, 2014 ) as the intervention (specially being in a group setting) could have elicited difficult internal events that activated experiential avoidance strategies. However, the participant reported increased "observing" on the mindfulness questionnaire. This appears contradictory to a self reported increase in experiential avoidance. One way of reconciling these findings is that the participant may have improved in his ability to observe internal events without cultivating greater psychological flexibility in responding to these experiences (as also found by Baer et al., 2006 in other samples). Consistent with this, the participant showed a decrease of the non-judgmental attitude after the intervention.

The C.MAP intervention aims to facilitate greater attunement to threatening psychotic experiences and internal events (including distressing thoughts, bodily sensations, emotions). Increasing awareness of these experiences without cultivating attitudes of acceptance and compassion may mean that these experiences will continue to feel threatening and trigger safety responses (such as experiential avoidance). Future studies should be careful to include measures of mindfulness, acceptance, and compassion to explore potential associations with increases or decreases in distress. Such an approach may provide a more fine-grained approach to identifying blocks to engaging in contextually based cognitive behavioural therapies and also provide a rationale for identifying adverse effects of intervention. Furthermore, it might be useful in future interventions to devote more time (this was a very brief intervention) to train the mindfulness and compassion skills in order to provide greater practice.

The participant showed no differences in external shame, but the levels of self-criticism decreased. On the other hand, the patient reported a higher capacity to self-soothe and calm. It is possible that the brief intervention tested was not long and powerful enough for these changes to be consolidated and to reflect an emotional change (shame feelings). Self-criticism is a defensive response to deal with feelings of shame (Gilbert, 2010), and although no other studies assessed selfcriticism, decreases in shame and self-criticism are essential in recovery and relapse prevention according to the social mentalities model for psychosis (Gumley et al., 2010). Furthermore, relapse has been associated with greater feelings of self-blame and shame (Gumley et al., 2006); therefore, intervention should focus on the activation of the safeness system (Gumley et al., 2010), which is essential for stress reduction and promotion of social bonding and affiliative behaviours (Gumley, Braehler, \& Macbeth, 2014; Gumley, Taylor, Schwannauer, \& Macbeth, 2014). 


\section{Limitations and Future Directions}

Some limitations should be taken into consideration. The study design and sample size (two case studies) and statistical analysis do not allow the generalisation of results for the target population. The lack of a control group (although such a small experimental sample would not allow valid comparisons) was also a limitation to be addressed in future studies. Therefore, our aim was not to prove efficacy but to illustrate the possible benefits that this integrative intervention could provide in psychosis and also motivate further clinical discussion about the possible benefits of the CCBT processes applied to psychosis. Regarding the assessment measures, one limitation of our study is the absence of a clinical interview to assess symptoms as an out- come measure and also as a part of the initial assessment of inclusion/exclusion criteria for participating in the study. The absence of a formal clinical assessment of cognitive deficits and difficulties in abstract thinking prior to selection for participation was an important limitation as it determined the final sample size. The first assessment session should have an important section devoted to motivate participants and to normalise difficulties (de-shaming). Participants found it difficult to complete the self-report measures, and future studies should also incorporate observer-based assessments. The absence of a follow-up assessment meant that we could not observe further changes in experiences over time. Although patients were encouraged to practice the exercises with a family member (and were given a detailed script), some participants reported difficulties in practicing exercises at home; future studies should make audio resources available to patients.

Other important considerations that future studies should take into account are the group effects on improvement (that could have influenced our results). A recent review has shown that non-specific effects (non-intervention-related) seem to occur in group therapy, with people diagnosed with schizophrenia in variables such as the improvement of negative symptoms and social functioning deficits (Orfanos, Banks, \& Priebe, 2015). Therefore, future studies should control this con- founding variable in their statistical analysis and make efforts to understand the mechanisms that might have contributed to efficacy (mediational and moderational analyses).

\section{Clinical Implications}

The present study developed a new brief clinical intervention that can be easily implemented and seems to have the potential to be adapted to different settings. The C.MAP, being brief and not very demanding (e.g., in terms of homework tasks, length of sessions, etc.), may be useful as a 
preliminary intervention for participants who refuse to engage in longer therapeutic pro- tocols. This integrative approach (based on compassion, mindfulness, and acceptance processes) may also be useful for this population in a non-directive way that provides different strategies that patients can try and select for themselves based on usefulness. Moreover, these emotion regulation strategies have been described as adaptive and beneficial in terms of stress reduction and promoting pro-social behaviours. Future studies using this integrated protocol could also explore the different contribution of these interrelated mechanisms in the therapeutic change process (component analysis).

The acceptability results indicate that the C.MAP was well tolerated by the participants (no adverse effects were reported), and in the two participants further analysed, it seems to have been beneficial to some extent.

Although clearly preliminary, future studies may continue to study C.MAP with larger samples and more sophisticated methods in order to understand the beneficial effects that this therapeutic approach can add to standard interventions (e.g., pharmacological).

\section{Acknowledgement}

We would like to acknowledge the collaboration of the Aveiro Mental Health Community Team (Psychiatry and Mental Health Department, Baixo Vouga Hospital Centre, Portugal) for which we are very grateful.

\section{References}

Abba, N., Chadwick, P., \& Stevenson, C. (2008). Responding mindfully to distressing psychosis: A grounded theory analysis. Psychotherapy Research, 18(1), 77-87. doi:10.1080/10503300701367992

Bach, P., Gaudiano, B., Hayes, S., \& Herbert, J. (2012). Acceptance and commitment therapy for psychosis: Intent to treat, hospitalization outcome and mediation by believability. Psychosis: Psychological, Social and Integrative Approaches, 5(2), 166-174. doi:10.1080/ 17522439.2012 .671349

Bach, P., \& Hayes, S. C. (2002). The use of acceptance and commitment therapy to prevent the rehospitalization of psychotic patients: A randomized controlled trial. Journal of Consulting and Clinical Psychology, 70, 1129-1139. doi:10.1037/0022-006X.70.5.1129

Bach, P., Hayes, S. C., \& Gallop, R. (2012). Long term effects of brief Acceptance and Commitment Therapy for psychosis. Behavior Modification, 36, 167-183. 
doi:10.1177/0145445511427193

Baer, R. A., Smith, G. T., Hopkins, J., Krietemeyer, J., \& Toney, L. (2006).

Using self-report assessment methods to explore facets of mindfulness. Assessment, 13(1), 2745. doi:10.1177/

1073191105283504

Barlow, D. (2002). Anxiety and its disorders: The nature and treatment of anxiety and panic (2nd ed.). New York, NY: Guilford.

Birchwood, M., Spencer, E., \& McGovern, D. (2000). Schizophrenia: Early warning signs. Advances in Psychiatric Treatment, 6, 93-101. doi:10.1192/apt.6.2.93

Birchwood, M., Trower, P., Brunet, K., Gilbert, P., Iqbal, Z., \& Jackson, C. (2007). Social anxiety and the shame of psychosis: A study in First Episode Psychosis. Behaviour Research and Therapy, 45, 1025-1037. doi:10.1016/j.brat.2006.07.011

Bond, F. W., Hayes, S. C., Baer, R. A., Carpenter, K. M., Guenole, N., Orcutt, H. K., ... Zettle, R. D. (2011). Preliminary psychometric properties of the Acceptance and Action Questionniare - II: A revised measure of psychological flexibility and experiential avoidance.

Behavior Therapy, 42(4), 676-688. doi:10.1016/j.beth.2011.03.007 Braehler, C., Gumley, A., Harper, J., Wallace, S., Norrie, J., \& Gilbert, P.

(2013). Exploring change processes in compassion focused therapy in psychosis: Results of a feasibility randomized controlled trial. British Journal of Clinical Psychology, 52(2), 199-214. doi:10.1111/bjc.12009

Castilho, P., \& Pinto-Gouveia, J. (2011). Auto-Criticismo: Estudo de validação da versão portuguesa da Escala das Formas do Auto- Criticismo e Auto-Tranquilização (FSCRS) e da Escala das Funções do Auto-Criticismo e Auto-Ataque (FSCS) [Self-criticism: Validation study of the Portuguese version of the Forms of Self Criticism and Reassurance Scale (FSCRS) and the Functions of self Criticism and Self Attack Scale (FSCS)]. Psychologica, 54, 63-86.

Chadwick, P., Hughes, S., Russel, D., Russel, I., \& Dagnan, D. (2009). Mindfulness groups for distressing voices and paranoia: A replication and randomized feasibility trial. Behavioural and Cognitive Psychotherapy, 37(4), 403-412. doi:10.1017/S1352465809990166

Chadwick, P., Newman-Taylor, K., \& Abba, N. (2005). Mindfulness groups for people with psychosis. Behavioural and Cognitive Psychotherapy, 33, 351-359. doi:10.1017/S1352465805002158

Chien, W. T., \& Lee, I. Y. (2013). The mindfulness-based psychoeducation program for Chinese patients with schizophrenia. Psychiatric Services, 64(4), 376-379. doi:10.1176/appi.ps.002092012

Chien, W. T., \& Thompson, D. R. (2014). Effects of a mindfulness-based psychoeducation 
programme for Chinese patients with schizophrenia: 2-Year follow-up. British Journal of Psychiatry, 205, 52-59. doi:10.1192/bjp.bp.113.134635

Freeman, D., Garety, P. A., Bebbington, P. E., Smith, B., Rollinson, R., Fowler, D., ... Dunn, G. (2005). Psychological investigation of the structure of paranoia in a non-clinical population. The British Journal of Psychiatry, 186, 427-435. doi:10.1192/bjp.186.5.427

García-Montes, J. M., Luciano, M. C., Hernández, M., \& Zalvivar, F. (2004). Aplicación de la Terapia de Aceptación y Compromiso (ACT) a sintomatología delirante: un estudio de caso [Application of the Acceptance and Commmitment Therapy (ACT) to delusion symptomatology: A case study]. Psicothema, 16, 117-124.

García-Montes, J. M., \& Pérez-Álvarez, M. (2001). ACT como tratamiento de síntomas psicóticos, El caso de las alucinaciones auditivas [ACT as a treatment to psychotic symptoms, the auditive hallucinations' case]. Análisis y Modificación de Conducta, 27, 455-472.

García-Montes, J. M., \& Pérez-Álvarez, M. (2010). Exposition in existential terms of a case of "Negative Schizophrenia" approached by means of Acceptance and Commitment Therapy. International Journal of Existential Psychology and Psychotherapy, 3(1), 1-18.

Gaudiano, B. A., Bush, A. M., Wenze, S. J., Nowlan, K., Epstein-Lubow, G., \& Miller, I. (2015). Acceptance-based behavior therapy for depression with psychosis: Results from a pilot feasibility randomized controlled trial. Journal of Psychiatric Practice, 21(5), 320-333. doi:10.1097/PRA.0000000000000092

Gaudiano, B. A., \& Herbert, J. D. (2006). Acute treatment of inpatients with psychotic symptoms using acceptance and commitment therapy: Pilot results. Behaviour Research and Therapy, 44, 415-437. doi:10.1016/j.brat.2005.02.007

Gaudiano, B. A., Herbert, J. D., \& Hayes, S. C. (2010). Is it the symptom or the relation to it? Investigating potential mediators of change in Acceptance and Commitment Therapy for psychosis. Behavior Therapy, 41, 543-554. doi:10.1016/j.beth.2010.03.001

Gilbert, P. (2010). Compassion focused therapy: Distinctive features. London, England: Routledge.

Gilbert, P., Clarke, M., Hempel, S., Miles, J. N. V., \& Irons, C. (2004). Forms and functions of selfcriticism and self-attacking: An exploration of differences in female students. British Journal of Clinical Psychology, 43, 31-50.

Gilbert, P., \& Procter, S. (2006). Compassionate mind training for people with high shame and self-criticism: Overview and pilot study of a group therapy approach. Clinical Psychology \& Psychotherapy, 13, 353-379. doi:10.1002/cpp.507

Goss, K., Gilbert, P., \& Allan, S. (1994). An exploration of shame measures: I: The 'other as 
shamer' scale. Personality and Individual Differences, 17, 713-717. doi:10.1016/0191-8869(94)90149$\mathrm{X}$

Gregório, S., \& Pinto-Gouveia, J. (2011). Facetas de Mindfulness: Características psicométricas de um instrumento de avaliação [Facets of mindfulness: Psychometric properties of an assessment instrument]. Psychologica, 54, 259-279.

Gumley, A., Braehler, C., Laithwaite, H., MacBeth, A., \& Gilbert, P. (2010). A compassion focused model of recovery after psychosis. International Journal of Cognitive Therapy, 3(2), 186-201. doi:10.1521/ ijct.2010.3.2.186

Gumley, A., Braehler, C., \& Macbeth, A. (2014). A meta-analysis and theoretical critique of oxytocin and psychosis: Prospects for attachment and compassion in promoting recovery. British Journal of Clinical Psychology, 53(1), 42-61. doi:10.1111/bjc.12041

Gumley, A., Karatzias, A., Power, K., Reilly, J., McNay, L., \& O’Grady, M. (2006). Early intervention for relapse in schizophrenia: Impact of cognitive behavioural therapy on negative beliefs about psychosis and self-esteem. British Journal of Clinical Psychology, 45, 247-260. doi:10.1348/014466505X49925

Gumley, A. I., Taylor, H. E. F., Schwannauer, M., \& MacBeth, A. (2014). A systematic review of attachment and psychosis: Measurement, construct validity and outcomes. Acta Psychiatrica Scandinavica, 129(4), 257-274. doi:10.1111/acps.12172

Hayes, S. C., Luoma, J., Bond, F., Masuda, A., \& Lillis, J. (2006). Acceptance and Commitment Therapy: Model, processes, and outcomes. Behaviour Research and Therapy, 44(1), 1-25. doi:10.1016/ j.brat.2005.06.006

Hayes, S. C., Strosahl, K., \& Wilson, K. G. (2011). Acceptance and commitment therapy. The process and practice of mindful change (2nd ed.). New York: Guilford Press. ISBN: 978-1-60918-9624.

Hayes, S. C., Villatte, M., Levin, M., \& Hildebrandt, M. (2011). Open, aware, and active: Contextual approaches as an emerging trend in the behavioral and cognitive therapies. Annual Review of Clinical Psychology, 7, 141-68. doi:10.1146/annurev-clinpsy-032210-104449

Jacobsen, P., Morris, E., Johns, L., \& Hodkinson, K. (2011). Mindfulness groups for psychosis; key issues for implementation on an inpatient unit. Behavioural and Cognitive Psychotherapy, 39, 349353. doi:10.1017/S1352465810000639

Jacobson, N. S., \& Truax, P. (1991). Clinical significance: A statistical approach to defining meaningful change in psychotherapy research. Journal of Consulting and Clinical Psychology, 59, 1219. doi:10.1037// 0022-006X.59.1.12 
Johnson, D., Penn, D., Fredrickson, B., Kring, A., Meyer, P., Catalino, L., \& Brantley, M. (2011). A pilot study of loving-kindness meditation for the negative symptoms of schizophrenia. Schizophrenia Research, 129, 137-140. doi:10.1016/j.schres.2011.02.015

Khoury, B., Lecomte, T., Comtois, G., \& Nicole, L. (2013). Third-wave strategies for emotion regulation in early psychosis: A pilot study. Early Intervention in Psychiatry, 9(1), 76-83. doi:10.1111/eip.12095

Laithwaite, H., O’Hanlon, M., Collins, P., Doyle, P., Abraham, L., Porter, S., \& Gumley, A. (2009). Recovery After Psychosis (RAP): A compassion focused programme for individuals residing in secure settings. Behavioural and Cognitive Psychotherapy, 37, 511-526. doi:10.1017/S1352465809990233

Langer, A. I., Cangas, A. J., Salcedo, E., \& Fuentes, B. (2012). Applying mindfulness therapy in a group of psychotic individuals: A controlled study. Behavioural and Cognitive Psychotherapy, 40(1), 105-9. doi:10.1017/S1352465811000464

Livingstone, K., Harper, S., \& Gillanders, D. (2009). An exploration of emotion regulation and in Psychosis. Clinical Psychology \& Psychotherapy, 16(5), 418-430. doi:10.1002/cpp.635

Lopes, B. (2010). Paranoia e Ansiedade Social na População Não-Clínica [Paranoia and social anxiety in non-clinical populations]. Doctoral thesis, University of Coimbra, Portugal.

Matos, M., Pinto-Gouveia, J., \& Gilbert, P. (2013). The effect of shame and shame memories on paranoid ideation and social anxiety. Clinical Psychology \& Psychotherapy, 20(4), 334-349. doi:10.1002/ cpp.1766

Matos, M., Pinto-Gouveia, J., Gilbert, P., Duarte, C., \& Figueiredo, C. (2015). The Other as Shamer Scale - 2: Development and validation of a short version of a measure of external shame. Personality and Individual Differences, 74, 6-11. doi:10.1016/j.paid.2014.09.037

Mayhew, S., \& Gilbert, P. (2008). Compassionate mind training with people who hear malevolent voices. A case series report. Clinical Psychology \& Psychotherapy, 15, 113-38. doi:10.1002/cpp.566

Newman-Taylor, K., Harper, S., \& Chadwick, P. (2009). Impact of mindfulness on cognition and affect in voice hearing: Evidence from two case studies. Behavioural and Cognitive Psychotherapy, 37(04), 397-402. doi:10.1017/S135246580999018X

Orfanos, S., Banks, C., \& Priebe, S. (2015). Are group psychotherapeutic treatments effective for patients with schizophrenia? A systematic review and meta-analysis. Psychotherapy and Psychosomatics, 84(4), 241-249. doi:10.1159/000377705

Pankey, J., \& Hayes, S. C. (2003). Acceptance and commitment therapy for psychosis. International Journal of Psychology and Psychological Therapy, 3, 311-328. 
Pinto-Gouveia, J., Gregório, S., Dinis, A., \& Xavier, A. (2012). Experiential avoidance in clinical and non-clinical samples: AAQ-II Portuguese version. International Journal of Psychology and Psychological Therapy, 12(2), 139-156.

Shawyer, F., Farhall, J., Mackinnon, A. J., Trauer, T., Sims, E., Ratcliff, K., ... Copolov, D. L. (2012). A randomised controlled trial of acceptance- based cognitive behavioural therapy for command hallucinations in psychotic disorders. Behaviour Research and Therapy, 50, 110-121. doi:10.1016/j.brat.2011.11.007

Tabak, N. T., \& Granholm, E. (2014). Mindful cognitive enhancement training for psychosis: A pilot study. Schizophrenia Research, 157, 312-313. doi:10.1016/j.schres.2014.06.002

Taylor, P. J., Gooding, P. A., Wood, A. M., Johnson, J., Pratt, D., \& Tarrier, N. (2010). Defeat and entrapment in schizophrenia: The relationship with suicidal ideation and positive psychotic symptoms. Psychiatry Research, 178, 244-248. doi:10.1016/j. psychres.2009.10.015

Udachina, A., Thewissen, V., Myin-Germeys, I., Fitzpatrick, S., O’kane, A., \& Bentall, R. P. (2009). Understanding the relationships between self-esteem, experiential avoidance, and paranoia: Structural equation modelling and experience sampling studies. Journal of Nervous and Mental Disorders, 197(9), 661-668. doi:10.1097/ NMD.0b013e3181b3b2ef

Van der Valk, R., Van de Waerdt, S., Meijer, C. J., Van den Hout, I., \& de Haan, L. (2013). Feasibility of mindfulness-based therapy in patients recovering from a first psychotic episode: A pilot study. Early Intervention in Psychiatry, 7(1), 64-70. doi:10.1111/j.1751- 7893.2012.00356.x

Veale, D., Gilbert, P., Wheatley, J., \& Naismith, I. (2014). A new therapeutic community: Development of a compassion-focussed and contextual behavioural environment. Clinical Psychology \& Psychotherapy, 22(4), 285-303. doi:10.1002/cpp.1897

White, R., Gumley, A., McTaggart, J., Ratrie, L., McConville, D., Cleare, S., \& Mitchell, G. (2011). A feasibility study of Acceptance and Commitment Therapy for emotional dysfunction following psychosis. Behaviour Research and Therapy, 49, 901-907. doi:10.1016/j. brat.2011.09.003

White, R. G., Gumley, A., McTaggart, J., Rattrie, L., McConville, D., Cleare, S., \& Mitchell, G. (2013). Depression and anxiety following psychosis: Associations with mindfulness and psychological flexibility. Behavioural and Cognitive Psychotherapy, 41(1), 4-51. doi:10.1017/ S1352465812000239

Wise, E. A. (2004). Methods for analyzing psychotherapy outcomes: A review of clinical significance, reliable change and recommendations for future directions. Journal of Personality Assessment, 82, 50-59. doi:10.1207/s15327752jpa8201_10

Zahra, D., \& Hedge, C. (2010). The reliable change index: Why isn't it more popular in academic psychology? Psychology Postgraduate Affairs Group Quarterly, 76, 14-19. 